ISSN: 0213-2052 - eISSN: 2530-4100

DOI: http://dx.doi.org/10.14201/shha201937529

\title{
ARISTÓTELES: LOS CIUDADANOS COMO JUECES Y EL CONCEPTO DE JUSTICIA GLOBAL ${ }^{1}$
}

\author{
Aristotle on Popular Juries and the Concept of Global \\ Justice
}

Elisabetta PODDIGHE

Universidad de Cagliari

poddighe@unica.it

Fecha de recepción: 8-04-2019; aceptación definitiva: 21-05-2019

ORCID: 0000-0001-6347-9432

RESUMEN: En este artículo se abordan las reflexiones que realizó Aristóteles en su Retórica y en su Política sobre el papel de los jurados populares y sobre los principios que establecen la función jurisdiccional de los ciudadanos jueces. Es en el marco de esta reflexión donde Aristóteles reconoce como principio básico de la función juzgante la necesidad de que la sentencia corresponda al èthos de la politeía y, por lo tanto, a esa noción de "justicia global", que es el idem sentire de la comunidad política.

Palabras clave: Aristóteles; jueces; justicia; ley; epieíkeia; politeía.

ABSTRACT: The article discusses Aristotle's thought as regards the role of popular juries and the principles that assigned judicial power to citizens. It is precisely within the framework of this reflection that Aristotle recognises the founding principle behind making judgements. This is the need for the sentence to correspond to the éthos of the constitution and therefore

1. El trabajo se ha realizado en el marco del proyecto HAR2015-63549-P (MINECO/ FEDER). 
to that notion of "global justice", the same feeling found in a political community.

Keywords: Aristotle; popular courts; justice; law; epieíkeia; politeía.

1. ARISTÓteles, la libertad política De los CiUdAdANOS Y EL DERECHO AL VOTO EN LOS TRIBUNALES

Aristóteles afirma en la Política que la libertad política de los ciudadanos se expresa principalmente en el ejercicio de voto en los tribunales. Por lo tanto, juzga positivamente la decisión atribuida a Solón de hacer al dêmos "dueño del voto" y, por tanto, "dueño del Estado" ". También en relación con esta medida atribuye Aristóteles a la reforma soloniana los contenidos de una protodemocracia: porque reconoce por primera vez al dêmos -entendido como la parte pobre y no aristocrática de los ciudadanos- la función jurisdiccional ${ }^{3}$.

Es necesario subrayar que la posición de Aristóteles está en sintonía con la expresada por otros escritores antiguos, tanto partidarios como contrarios a la democracia, que por lo general parecen proclives a juzgar como propio de la mejor constitución el principio según el cual la función jurisdiccional es ejercida por todos cuando la decisión se refiere a asuntos de interés público ${ }^{4}$.

El principio de la participación ciudadana directa en la función judicial -la krísis- es un principio "fundamental para la propia condición de ciudadano"s. Se trata, en efecto, de una función soberana por cumplirse "indefinidamente ${ }^{6}$. Y cuando quien la ejerce es el dêmos en su totalidad, esa función es incluso rasgo distintivo de la democracia ${ }^{7}$.

Por otro lado, el establecimiento de jurados populares parece ser una buena solución para el problema de la administración de justicia incluso

2. Pol. 1273b 35-1274a 21, 1281b 32-34; Ath. Pol. 9.2; cfr. Mirhady, 2006, p. 3; Osborne, 2006, pp. 10 ss.; Cammack, 2013, p. 93 ss.; Poddighe, 2014, pp. 102 ss., 186 ss., 200 ss.; Pelloso, 2017, pp. 518-556; Loddo, 2018, pp. 113-118; SáncheZ Madrid, 2018, pp. 38 ss.

3. Talamanca, 1994, pp. 22-7; Mirhady, 2006, p. 4; López Barja de Quiroga, 2009, 212 ss.; CAMmack, 2013, pp. 53 ss., 92 ss.; Lane, 2013; para esta acepción de dêmos cf. Poddighe, 2014, p. 122.

4. Cf. Maffi, 2004, p. 308; Mirhady, 2006, p. 9; Ventura, 2009, p. 227.

5. Plat. Nom. 768b; Arist. Pol. 1275 a 22-23, 1275b 18-19; YaCK, 1993, p. 189; MafFI, 2004, p. 306; Mirhady, 2006, pp. 5-6; Osborne, 2006, p. 10 ss.; López Barja de Quiroga de Quiroga, 2009, pp. 209 ss.; CAMMACK, 2013, pp. 53 ss., 90 ss.

6. Poddighe, 2014, pp. 142 y 202.

7. Rhet. $1365 \mathrm{~b} \mathrm{31-32.}$ 
en regímenes políticos distintos a la democracia, como lo demuestran las reflexiones, convergentes desde este punto de vista, de Platón en las Leyes ${ }^{8}$ y Aristóteles en la Política $a^{9}$. Ser juzgado por muchos en lugar de por unos pocos es preferible en todos aquellos casos de relevancia pública en los que la ley no decide ${ }^{10}$, pues entonces, como observa Aristóteles, parece no solo preferible, sino más justo que la krísis sea colectiva, es decir, que la función judicial se confíe a los muchos ${ }^{11}$.

Como trasfondo se halla el gran problema de las prescripciones generales, que no se pueden prever todos los casos individuales de la práxis a los que se deben adaptarse en cada ocasión ${ }^{12}$. En este segundo caso, la responsabilidad del control se confía a los "muchos". Como trasfondo se encuentran también los niveles de competencia asignados en el campo de la legislación: la sabiduría legislativa, -que es una prerrogativa de los nomóthetai-, y la sabiduría política asignada a los ciudadanos que son responsables de la aplicación de las leyes ${ }^{13}$. La justificación teórica de esta jerarquía es clara en la obra aristotélica y su posición respecto a este punto se expresa de manera neta no solo en la Política, sino también en la Retórica y en la Ética a Nicómaco: la legislación relativa a los universales es materia para unos pocos, mientras que son los muchos los que actúan decidiendo los casos individuales de la práxis ${ }^{14}$.

El papel de la colectividad -que aparece excluido de la acción de la reforma legislativa- se contempla en cambio en el ámbito de aplicación de las leyes a casos particulares: aquí la acción de los "muchos" interviene para juzgar y decidir los casos particulares que la ley no puede decidir o no puede decidir bien ( $P o l$. 1287a 19-28, cfr. 1282b 2-10).

En el tercer libro de Política, el problema se aborda con referencia particular al problema de las figuras institucionales a las que debe confiarse el control de la adaptación de la ley a los casos particulares (1286a 10-31; 1287a 19-28). Aristóteles considera que "las leyes expresan solo indicaciones generales y carecen de valor normativo para casos individuales"

8. Nom. 768a-b; Faraguna, 2007, p. 92.

9. Talamanca, 1994, p. 74; Maffi, 2004, p. 305; Mirhady, 2006, pp. 5-6.

10. EN 1137b 14-19; Pol. 1287b 15-18; Rhet. 1354a 26-b 10.

11. Pol. 1286a 21-40, 1287b 8-29, 1281b 1-25, 1282a 14-30. Mirhady, 2006, pp. 6-8; SANCHO Rocher, 2007, pp. 143; CAMmaCK, 2013, pp. 53 ss., 90 ss.

12. Aubenque, 1965, pp. 109-113; Harris, 2013, pp. 177-182; Cfr. Bertelli, 1989, p. 311

13. Leszl, 1989, pp. 124-126; YACK, 1993, pp. 190-193; BulLen, 1997, pp. 229-241; Mayhew, 2009, pp. 531-535; Bertelli, 2015, pp. 21-23; Poddighe, 2018 b.

14. Rhet. 1354a 31-b16; EN 1141b 21-29; Pol. 1292a 32-37. Para la discusión de los pasajes cfr. Bullen, 1997, pp. 229-241. Cf. Wormuth, 1979, pp. 17-19; Simpson, 1998, pp. 308-309; MaYhew, 2009, pp. 533-534; Harris, 2013, pp. 177-182; Bertelli, 2015, pp. 21-26. 
y que "por esta razón, evidentemente, la mejor constitución no será la que se atiene a la letra de las leyes" y las leyes no deben ser "soberanas para lo que cae fuera de su alcance" (1286a 10-24). En este contexto, Aristóteles aborda el problema de la responsabilidad de quienes deben decidir preguntándose: «En todo lo que no es posible que la ley juzgue en absoluto o que lo juzgue bien, ¿debe gobernar uno solo, el mejor, o todos?” (1286a 2425). La solución aristotélica no debe sorprendernos. De hecho, Aristóteles trae a colación el ejemplo de los organismos colectivos cuyas decisiones tienen siempre que ver con casos particulares y que parece poder identificar con la asamblea, el consejo, los tribunales (1286a 26-28) ${ }^{15}$.

Los ciudadanos reunidos en asamblea o en los tribunales judiciales -a quienes se encomienda la administración de la práxis- tendrán que ejercer en esas sedes el control sobre el cambio de las leyes. Pero ¿de qué manera? Aristóteles especifica que su intervención se contempla en los casos en que la ley no puede decidir, por lo tanto, en el ámbito de "lo que no es posible que la ley juzgue en absoluto o que juzgue bien" (1286a 24-25), en los casos en los "que la ley parece no poder definir" (1287a 24) y en asuntos sobre los cuales "es incapaz de precisar" $(1287 \mathrm{~b} 22-23)^{16}$. Las decisiones de muchos "se refieren todas a casos concretos" (1286a 27-28) y solo para estos casos es oportuno que sea la multitud la que decida porque ajuzga mejor muchas veces una multitud que un individuo cualquiera" (1286a 3031) porque en estos casos este no "podría decidir" (1287a 24-25) ${ }^{17}$.

En este contexto, la acción colectiva se expresa a través de los instrumentos más adecuados para regular los casos particulares de la práxis, en la asamblea y en los tribunales. En el primer caso, la acción política de los ciudadanos se expresa a través de decretos, que son distintos de las leyes precisamente por el carácter particular de los primeros y general de las segundas. La distinción entre leyes (nómoi) y decretos (psēphismata)

15. "En este momento son estos últimos quienes, reunidos en asamblea (sunióntes), administran la justicia (dikázousin), deciden (bouleúontai) y deliberan (krínousin), pero todas estas deliberaciones (kriseis) se refieren a casos individuales". Cf. Accattino, 2013, p. 225. LANE, 2013, pp. 262-263, propone leer en el pasaje una referencia al papel político de los jueces populares

16. Aubenque, 1965, p. 111ss. Cfr. también Pol. 1282b 3-5 sobre los archai que debe hacer valer su autoridad en casos donde las leyes no pueden formularse con precisión.

17. Cf. también Pol. 1281a 42-1281b 2: "los más, cada uno de los cuales es un hombre mediocre, pueden, sin embargo, reunidos, ser mejores que aquéllos, no individualmente, sino en conjunto". Véase por el contrario, Platón en Política (294a-297b) y en las Leyes (VI 769d-e; 875d-876a). Cf. Aubenque 1965, pp. 109-113; Schütrumpf 1991, pp. 278-279; Camassa 2003, p. 162; Pezzoli 2012, p. 295ss; Accattino 2013, p. 224ss. Sobre las condiciones históricas en que se mueve Aristóteles son particularmente útiles las reflexiones de Moraux 1965, pp. 140-157. 
-recurrente en la obra política aristotélica ${ }^{18}$ - tiene como base la ya considerada e "infranqueable" diferencia entre legislación, "que es cometido de figuras especiales que actúan en circunstancias especiales y hacen un trabajo que no puede y no debe ser modificado", y administración, "que forma parte de los asuntos cotidianos de la polis" "19. En este ámbito, el control del cambio de las leyes debe entenderse como un auténtico freno para la actividad de corrección de las leyes. Cuando la ley no puede regular el caso en particular, interviene el decreto que, siendo más dúctil ${ }^{20}$ y flexible que la ley "se adapta a los hechos" (EN 1137 12-33)

En cambio, en relación con los tribunales, Aristóteles afirma que la ley encarga a los magistrados (toùs árkhontas) ujuzgar y administrar las demás cosas con el criterio más justo" y "además, les permite rectificarla en lo que, por experiencia, les parezca que es mejor que lo establecido" (1287a 25-28). Aristóteles no menciona aquí “el criterio más justo" -la gnōmé dikaiotáté- para ejemplificar su razonamiento teórico con un caso concreto, el ateniense, por más que los comentaristas del pasaje aristotélico sugieren acertadamente la comparación con el juramento de los heliastas ${ }^{22}$. De hecho, en Atenas los jueces juraban "votar de acuerdo con las leyes en los asuntos en los que existen leyes; en asuntos en los que no las hay, votar con el juicio más justo" (Poll VIII, 5-7)23. El juramento heliástico, sin embargo, no es el tema que centra la discusión aristotélica. La referencia al criterio "más justo", parece tener la función de rematar su razonamiento sobre el tema de la adaptación de la ley a los casos

18. Rhet. 1354a 31-b16; EN 1137b 12-33 e 1141b 21-29; Pol. 1292a 4-8, 13-37. cf. Aubenque, 1965, pp. 109-113; BRunschWig, 1980, pp. 518, 524 ss.; Bullen, 1997, pp. 229241; Kraut, 2002, pp. 105-106; Frank, 2005, pp. 115-126; WeXler-Irvine, 2006, pp. 116-134; Accattino, 2013, p. 225; Pezzoli, 2014a, pp. 169-170; Bertelli, 2015, p. 21; Bearzot, 2016, pp. $95-98$

19. Canevaro, 2014, p. 287

20. Cf. Frank, 2005, p. 114 n. 10 sobre la naturaleza estática de las leyes que les impide adaptarse a situaciones concretas. Son particularmente útiles las reflexiones de Campeggiani, 2009, pp. 293-300.

21. EN 1137b 12-33. Cf. Aubenque, 1965, pp. 111-112, p. 156; Moraux, 1965, pp. 136140, pp. 141ss., pp. 156-57; WORMUTH, 1978, p. 19; YACK, 1993, pp. 198-199; VERGNIÈRES, 1995, p. 215; Bullen, 1997, pp. 231-237; Wexler-Irvine, 2006, pp. 116-134; CAMPegGiani, 2009, pp. 293-300; BEARZOT, 2016, pp. 95-98.

22. Cf. Wexler-Irvine, 2006, p. 133; Accattino, 2013, p. 231; Horn, 2013, p. 242; Lord, 2013, p. 93.

23. Reseña de fuentes y estudios en BEARZot, 2013, pp. 85-98; Harris, 2013, pp. 274301; Poddighe, 2014, pp. 272-298. 
particulares realizados por los jurados populares como corrección no permanente de la letra de la ley ${ }^{24}$.

Que quien juzgara sobre asuntos de interés público fuese «la opinión pública" era, en definitiva, una idea totalmente aceptada por Aristóteles ${ }^{25}$. Y, por lo tanto, el sistema judicial ateniense, que confiaba a grupos más o menos numerosos de jurados el derecho y el poder de juzgar sobre esos asuntos, parece ser reconocido por Aristóteles como no solo legítimo, sino como indispensable.

Otra razón por la cual el sistema de jurado popular no es para Aristóteles criticable en sí mismo ha de buscarse en el hecho de que la extensión al pueblo del derecho a administrar justicia representaba para los antiguos un principio constitutivo de la primera democracia: la que se debe a la reforma de Solón. A Solón, a su idea democrática, se remitía la decisión de asignar al pueblo el derecho a decidir sobre asuntos de relevancia pública (sea cual sea nuestra interpretación del carácter originario de ese tribunal) ${ }^{26}$. Hay un testimonio de Aristóteles particularmente indicativo de la posición específica adoptada por el filósofo con respecto a esta generalizada percepción antigua. Contra las críticas de quienes vinculaban la degeneración de la democracia de los siglos v y IV a la primera institución de los tribunales populares, Aristóteles objeta que los desarrollos posteriores no son imputables a esa primera decisión. Si acaso, habría que juzgarlos más bien como consecuencia del hecho de que, después de la reforma de Efialtes, el poder de los jurados populares dejó de operar con el freno de las medidas correctivas previstas por Solón. De ahí la invitación de Aristóteles a los detractores de la acción soloniana para que evaluaran su reforma de forma global (medidas correctivas incluidas), en vez de referirse a disposiciones concretas ${ }^{27}$.

Aristóteles tampoco considera problemática la voluntad de Solón de inducir al dêmos a familiarizarse con la interpretación de las leyes ${ }^{28}$. En los asuntos públicos, como hemos visto, la interpretación de los muchos es preferible a la de unos pocos, siempre que podamos contar con límites

24. Sobre la reflexión aristotélica sobre el tema de los cambios en la ley cfr. ahora PodDighe, 2018b.

25. Maffi, 2004, p. 305; Mirhady, 2006, p. 9; SANCho Rocher, 2008, pp. 939-940; López Barja de Quiroga, 2009, pp. 204 ss.; Ventura, 2009, p. 227.

26. Sea que pensemos en dikasteria seleccionados mediante sorteo (HANSEN, 2001, pp. 186 ss.), o en las sesiones judiciales de la asamblea popular (RHODES, 1981, p. 160, Ostwald, 1986, pp. 10-1) o el Consejo de los Cuatrocientos (Valdés Guía, 2008). Cfr. PodDighe, 2014, pp. 139-150; 192-203.

27. Ath. Pol. 9.2. Poddighe, 2018a, pp. 147-174.

28. Ath. Pol. 9.2. 
claros: por ejemplo, el hecho de que el poder de los jurados populares se delimitara frente a otras instituciones judiciales encargadas de decidir casos de particular relevancia y cuyas sentencias eran definitivas ${ }^{29}$.

En cuanto rasgo original y constitutivo de la mejor forma de democracia, el derecho del pueblo constituido en tribunal para interpretar el texto de las leyes, por lo tanto, no era criticable en sí mismo. El hecho de que Solón hubiera atenuado sus disposiciones en ámbito judicial al prever la activación o el fortalecimiento de adecuadas funciones de control -las llamadas competencias "añadidas" (epitheta) del Areópago ${ }^{30}$ - legitimaba, a juicio de Aristóteles, la disposición que extendía a todo el dêmos el ejercicio de la función jurisdiccional ${ }^{31}$.

En este marco, es comprensible que Aristóteles y otros teóricos antiguos no se hayan planteado el problema de definir el estatus de los jueces y no hayan atribuido en clave teórica un valor ideológico particular a la distinción entre tribunales compuestos por magistrados y tribunales compuestos por ciudadanos ${ }^{32}$. Una distinción que los teóricos antiguos consideraron solo en relación con la naturaleza pública o privada de los asuntos que se juzgaban: mientras que en el segundo caso lo correcto es que decidan los magistrados ${ }^{33}$, con referencia al primero lo es que los jueces sean seleccionados entre todos. En efecto, todos deben ejercer control sobre las instituciones, sobre la acción de los magistrados, sobre los patrimonios ${ }^{34}$. Son estas cuestiones que Aristóteles considera de la mayor importancia, porque las decisiones erróneas sobre estos asuntos pueden inducir a metamorfosis constitucionales ${ }^{35}$.

Con todo, incluso cuando la reflexión se centra en el tema de la emisión de sentencias, de la relevancia política que estas pueden adquirir, Aristóteles no hace de la competencia y capacitación del juez objeto de una reflexión teórica profunda ${ }^{36}$. El problema no es "quién juzga". En todo caso, será el de asegurar que quienes juzgan estén obligados a hacerlo

29. MacDowell, 1978, p. 39; Ostwald, 1986, p. 48; Wallace, 1989, p. 55; Talamanca, 1994, p. 24.

30. Poddighe, 2014, pp. 192-203, 331-335

31. Para la especificidad de esta reconstrucción en comparación con la propuesta por los atidógrafos cf. Poddighe, 2014.

32. MAFFI, 2004, p. 308.

33. Maffi, 2004, p. 308; Mirhady, 2006, p. 9; Sancho Rocher, 2007, p. 146; Ventura, 2009, p. 227.

34. Pol. 1317b 25-28, con Mirhady, 2006, pp. 9-12, y Ventura, 2009, pp. 227 ss.

35. Pol. 1300b 36-38.

36. Maffi, 2004, p. 308. La educación de los jueces convocados para resolver casos que la ley no decide (Pol. 1287a 25-1287b 25) se mide en relación con sus cualidades éticas, no profesionales (Саммаск, 2013, pp. 92 ss.). 
respetando la ley, es decir, bajo el control de quienes supervisan el cumplimiento de las leyes. La cuestión relevante es, en definitiva, vigilar la emisión de las sentencias para limitar la discrecionalidad de los jueces.

Con respecto a la atención que Aristóteles prestó a las "modalidades para dictar sentencias", se ha observado acertadamente que la reflexión aristotélica muestra que es consciente de los aspectos críticos de un sistema procesal en el que, como en Atenas, la sentencia del juez se basa en su libre convencimiento y por lo tanto "el juez está obligado a jurar para dar efectividad a la sentencia, ${ }^{37}$.

Aristóteles aborda el problema en su Retórica, cuando reflexiona sobre la primacía de la ley, que debería limitar (como sucede en el Areópago) la discrecionalidad de los jueces, y sobre la "mejor facultad de juicio" que fundamenta la libre interpretación de los jueces populares ${ }^{38}$.

¿Cuáles son los rasgos sobresalientes de la reflexión aristotélica?

El dato más relevante es que Aristóteles, no diferenciándose de otros teóricos antiguos, no juzga la criticidad de este aspecto en relación con la ausencia de un derecho codificado.

Una rápida comparación con la reflexión moderna sobre la institución de los jurados populares puede ayudarnos a reconocer la especificidad de la posición adoptada sobre el tema por Aristóteles y por los principales teóricos antiguos.

Leyendo las páginas que el historiador de derecho romano Giuliano Crifò ha dedicado a la institución del jurado popular, a su "continuidad entre la experiencia romana y la moderna", queda claro de inmediato por qué ha sido la experiencia romana y no la griega la que ha representado la "gramática política" de los principales teóricos de la institución moderna del jurado popular (de Montesquieu a Rousseau, de Blackstone a Filangieri) ${ }^{39}$. Lo que faltaba en la experiencia griega era un derecho formulado positivamente que hiciera la competencia de decisión de los jurados populares más técnica y menos discrecional. En particular, se echa en falta una elaboración técnico-jurídica del dato normativo que fuera punto de referencia imprescindible y freno indispensable de los jueces populares ${ }^{40}$. En resumen, se echa en falta la posibilidad de frenar y regular este poder

37. Maffi, 2004, pp. 309-11. Para una revisión de las fuentes y estudios sobre el juramento heliástico, cfr. Bearzot, 2013, pp. 85-98; Harris, 2013, pp. 274-301; Poddighe, 2014, pp. 272-298.

38. Rhet. 1354a 24-1375a 3; 1375a 30-1375b 21; MAFFI, 2004, p. 314.

39. CRIFÒ, 2005, pp. 52-5. Útil también la perspectiva comparatista de LóPEZ BARJA DE Quiroga, 1995, pp. 138-152.

40. Talamanca, 1994, pp. 19-32. 
político, que solo está representado por la «subordinación del derecho a juzgar de los jurados" a la operatividad de un derecho formulado positivamente.

Por el contrario, Aristóteles y los principales teóricos políticos griegos demuestran que no consideraron el problema político de la participación popular en la administración de justicia en relación con las cuestiones críticas que parecen evidentes para un historiador jurídico moderno. Y ello no porque subestimaran el problema de la formulación de la ley, sino porque la mentalidad jurídica griega admitía la posibilidad de que la ley fuera incapaz de especificar el delito, que de hecho podría definirse durante el proceso $^{41}$.

Se trata de una posición que, al menos en el caso de Aristóteles, puede reconocerse con relativa claridad.

Ha de señalarse, de forma preliminar, que, en el marco de la reflexión que Aristóteles reserva al problema de la emisión de las sentencias, la importancia atribuida al tema de la formulación de la ley no es irrelevante.

Por lo tanto, si bien resulta correcto llegar a la conclusión de que la posibilidad de "formular leyes más precisas y fundamentadas a nivel sustantivo" no representa para Aristóteles la solución al problema de limitar la discrecionalidad de los jueces para determinar la sentencia ${ }^{42}$, es útil en todo caso dar cuenta de la forma con la que el filósofo reflexiona sobre este tema y llega a reconocer los límites de la ley.

Debemos comenzar por el hecho de que Aristóteles asignaba a la "Ciencia política" entendida como ciencia práctica el objetivo de formar un legislador capaz de formular la ley de la mejor manera posible con respecto a la definición del delito, es decir, un legislador consciente de esta criticidad $^{43}$. El proceso, observa Aristóteles, debe resolverse mediante leyes positivas, que deben constituir en la medida de lo posible la base del juicio de los jueces, de acuerdo con lo que ocurre en el Areópago ${ }^{44}$. Las leyes correctamente establecidas deben, hasta donde sea posible, determinar

41. Paoli, 1933, pp. 58, 87; Wallace, 2006, p. 421; Sancho Rocher, 2007, p. 145; Osborne, 2010, pp. 171-204; Poddighe, 2014, pp. 277-294, 295-303.

42. MAFFI, 2004, p. 314.

43. Bodéús, 1991; Ventura, 2009; Osborne, 2010, p. 204. El legislador tiene la tarea de lograr el mayor bien para la comunidad ( $E N 1094 \mathrm{~b} 4$ ss.), y esto gracias a un cuerpo de leyes coherentes con el fin de la politeía, que es la buena vida de los ciudadanos (Pol. 1280b 40-41), quienes deberán observar los dictados de las leyes (EN 1102a 7-9). Con miras a estabilizar el marco constitucional y legislativo, es necesario sobre todo saber (para evitarlo) lo que orienta la constitución hacia el cambio. Cfr. Poddighe, 2014, pp. 35-38, 66-68; Poddighe, 2018b.

44. Rhet. 1354a 23-24; SANCHO Rocher, 2007, pp. 147 ss., 159 ss. 
todos los casos y dejar lo menos posible al arbitrio de los que juzgan. La determinación del delito, prosigue en efecto Aristóteles, corresponde a unos pocos de buen sentido y capaces de legislar (los legisladores) en lugar de los muchos (los jueces), por lo que no puede dejarse en manos de la sentencia "de un modo imprevisto" y, en cambio, debe tener lugar "luego de haber deliberado mucho tiempo", es decir, tras largas reflexiones del legislador ${ }^{45}$.

Los problemas que señala la práctica judicial en relación a la incapacidad de las leyes positivas para determinar todos los casos parecen ser bien conocidos por Aristóteles. Es frecuente -observa Aristóteles en la Retórica- que "quienes reconocen haber realizado la acción no reconozcan, en cambio, sea su calificación, sea a que se refiere esa calificación, pues, por ejemplo, reconocen haber tomado, pero no robado; haber golpeado primero, pero no ultrajado" ${ }^{46}$ : cuando en definitiva se mira la "letra" y no la "inteligencia" del legislador ${ }^{47}$. El caso paradigmático es el que se lee en el Contra Teomesto de Lisias, donde el acusado se defiende afirmando que su crimen "no está entre las expresiones prohibidas" y el actor afirma que "la discusión ha de versar no sobre las palabras, sino sobre su significado" ya que "hubiera sido un enorme trabajo para el legislador escribir todas las palabras que tienen el mismo significado" ${ }^{48}$.

En efecto, el juicio de Aristóteles llega a la misma conclusión que el protagonista del discurso de Lisias: cabe esperar que la formulación de la ley sea capaz de definir tantos casos como sea posible (y este es de hecho el objetivo de la actividad del legislador), pero es imposible que el legislador llegue a contemplar todos los casos concretos. "Se le pasaría la vida a quien intentase la enumeración de todas las posibilidades ${ }^{49}$, dice Aristóteles, constatando que incluso el mejor legislador no puede prever todos los casos de aplicación de su norma, sino solo "la mayor parte".

Que no eran resolutorios, respecto a esta dificultad, los procesos de revisión y reformulación de leyes -como aquellos a los que fueron sometidas las leyes de Solón a fines de siglo $\mathrm{v}^{50}-$, nos lo sugiere el hecho de que los modernos creen que, al final de esas revisiones, la definición del delito

45. Rhet. $1354 \mathrm{a} 33-1354 \mathrm{~b} 5$.

46. Rhet. 1374 a $1-5$.

47. Rhet. $1374 \mathrm{~b} 13$.

48. Lys. 10.6-16; TodD, 1996, p. 122; SANCHO Rocher, 2007, p. 166.

49. Rhet. 1374 a 33.

50. En un intento por llegar a una formulación menos susceptible a diferentes interpretaciones, las leyes de Solón fueron sometidas a dos procesos de revisión, por parte de un colegio de anagrapheis en el 410-409 (Lys. 30.2-3) y nuevamente desde el 403 (y 1.81-84; Arist. Ath. Pol. 35.2; Schol. And. 1.39) al 399 (Lys. 30.4); Cfr. RHodes, 1991; 
no parecía mejor pormenorizada ${ }^{51}$. Además, es igualmente indicativo, en este sentido, que el tema de la revisión no sea objeto de reflexión teórica ${ }^{52}$ y que, cuando se toma en consideración, no se le otorgue un desarrollo significativo ${ }^{53}$.

¿Qué podría, entonces, situar a los jueces en condiciones de ejercer su mejor facultad de juicio?

La reflexión aristotélica parece dar relieve especialmente a dos medidas que interactúan entre sí para contener la discrecionalidad de los jueces y que deberían ser contempladas en su conjunto por una recta constitución o politeía. La primera es prever funciones de vigilancia sobre la correcta aplicación de las leyes por parte de los jueces, en los casos en los que los delitos que se juzgan estén adecuadamente definidos por las leyes escritas. En lo que se refiere a esta primera medida, la posición aristotélica se reconoce en el marco de la reflexión reservada a la cuestión del control de las leyes, confiada al Areópago hasta el 462, y luego transferida por Efialtes y Pericles a los órganos democráticos de toma de decisiones. Un tema que he tratado ampliamente en otro lugar ${ }^{54}$.

La segunda medida, sobre la que me gustaría detenerme aquí, es la que se activa cuando la politeia es capaz de formar ciudadanos educados de acuerdo con sus principios y, por lo tanto, en condiciones de juzgar según el sentido común (epieíkeia) y en conformidad con las leyes no escritas tanto delitos no determinados por la ley escrita como delitos que, aunque contemplados por la ley escrita, requieren un ejercicio de sentido común "que está fuera de la ley escrita " $^{55}$ : en todos esos casos, una educación coherente con el èthos de la politeía permite al juez ejercer la mejor facultad de juicio ${ }^{56}$.

Volonaki, 2001; SANChO Rocher, 2007-2008; Rhodes, 2011, pp. 18-9; SHear, 2011, pp. 79111; Poddighe, 2014, pp. 127-139.

51. Poddighe, 2014, pp. 277-294, 295-303.

52. Yack, 1993, p. 187 n 33; Boegehold, 1996, p. 210. Rápido también el juicio de Platón (Nom. 769d-e, 772a-d). Cf. PodDighe, 2018b.

53. Pol. 1268b 26-1269a 20.

54. Para esta discusión remito a PodDighe, 2014, pp. 245-258, 331-335. Sobre el asunto véase ahora SÁNCHEZ MADRID, 2018, pp. 48 ss. Niega valor a la reconstrucción aristotélica ZACCARINI, 2018.

55. Rhet. 1374 a 28-29.

56. Sobre la importancia de conocer el carácter o èthos de una constitución, cf. Rhet. 1365b 21-1366a 21. Sobre el peso de ese argumento en la práctica oratoria, cf. WaLlace, 2007, p. 187; Poddighe, 2014, p. 59 ss y 273 ss; Ead. 2018a, pp. 158-161; Canevaro, 2018, pp. 5-29. 


\section{ARISTÓteles y EL CONCEPTO DE JUSTICIA GLOBAL}

La posición de Aristóteles se define en la Retórica, en el marco de reflexión dedicado a los criterios que deben regir el dictado de las sentencias por parte de los jurados. De lo que se trata es de la interpretación de los jueces en relación con lo que se ha definido a menudo como la open texture de la ley, es decir, su carácter "abierto" ${ }^{57}$ : una característica que obliga al jurado a interpretar la ley de acuerdo con su mejor juicio.

El problema que Aristóteles aborda es el siguiente: ¿sobre qué principios debe el juez determinar su mejor facultad de juicio?

El dato que debe destacarse es la compatibilidad de la reflexión aristotélica, tal como se desarrolla en la Retórica, con la práctica judicial atenien$\mathrm{se}^{58}$. Una compatibilidad que ha de reconocerse sea cual sea la valoración de la naturaleza de ese tratado: "a work of theory" como lo ha definido Harris $^{59} \mathrm{o}$ "un traité essentiellement pratique", como cree Bertrand ${ }^{60}$.

Varios elementos resultan indicativos a este respecto. En primer lugar, el hecho de que los escritores atenienses identificaran a menudo como un rasgo propio del sistema dicástico la dialéctica entre la ley positiva y el "sentido común" del jurado, entre nómos y tò epieikés. Los testimonios de Aristófanes, al igual que los del Viejo Oligarca o de Iseo, atestiguan que el tema de la libre convicción de los jueces basada en el principio de conveniencia y de sentido común -la epieíkeia- se identificaba como el rasgo distintivo del sistema de jurados populares atenienses ${ }^{61}$. De manera similar, el retórico Gorgias celebraba la mentalidad jurídica ateniense en cuanto capaz de emanciparse de la rigidez de la ley y de fundarse en el "sentido común" (DK, 82 B6) $)^{62}$.

En este contexto, es indicativo el hecho de que Platón, cuando teoriza en las Leyes un cuerpo de jurados que representa exactamente lo contrario de los jurados populares atenienses -se trata en efecto de magistrados sometidos a rendición de cuentas y capaces de decidir la pena con total

57. Millett, 1990, p. 179, lo define como "laxity", Farenga (2006, p. 331), en cambio, como "negociabilidad". Véase también Johnstone, 1999, pp. 24-33, Harris, 2000, pp. 27 ss., LanNi, 2006, pp. 72 ss., Wallace, 2006, pp. 422-3.

58. Talamanca, 1984 , p. 338; Sancho Rocher, 2007, p. 147; Harris, 2013, pp. 36-41, 47.

59. HarRis, 2006, p. 162. El tratamiento más detallado de esta posición es el de MeYerLaurin (1965, pp. 49 ss.); cfr. también Carey, 1996, pp. 36-9, Johnstone, 1999, p. 149 n. 97, O'Neil, 2001, pp. 22-3, Talamanca, 2008, p. 72 (pero véase Talamanca, 1994, p. 44).

60. Bertrand, 2002, p. 184; cfr. también Sancho Rocher, 2007, p. 153; Kasai, 2010, p. 124.

61. Poddighe, 2014, pp. 277-303.

62. Poddighe, 2014, pp. 277-294. 
autonomía respecto a las exigencias de las partes en causa (Plat, Nom. 761e, 766d-768c, 876b) ${ }^{63}$-, no tome en consideración el sentido común o la epieíkeia como la base de su juicio ${ }^{64}$.

El corpus de los oradores áticos -los estudios son claros a este respecto- confirma la operatividad de esta dialéctica ${ }^{65}$. De manera particular Iseo, que basa sus argumentos a menudo en el sentido común y en contra de la ley ${ }^{66}$, pero también Demóstenes, que también admite esta función del juez, cuando observa que más que la letra de la ley lo que cuenta es la preocupación del legislador (Dem 20.102, 22.30) ${ }^{67}$.

¿Cuál es pues la posición de Aristóteles? Con respecto a la interacción entre nómos y tò epieikés, entre ley y sentido común del juez, el problema que Aristóteles aborda en la Retórica es precisamente la dialéctica reconocible entre las principales tareas encomendadas al juez: la tarea de interpretar la ley y la de definir con "su" sentencia la noción de lo "justo legal" (lo dikaion).

La reflexión de Aristóteles llega a situar ambas funciones del juez dentro del espacio operativo de la epieíkeia. Hay tres momentos destacados en su reflexión. Es necesario partir del problema planteado por la open texture de la ley, que para Aristóteles es la incapacidad del legislador para definir todos los casos. El dictado de la ley -observa el filósofo- no siempre es capaz de regular el caso concreto (Rhet. 1374a 1 ss., Pol. 1287b 19-20). Esto se debe a que lo particular puede escapársele al legislador o a que el legislador debe necesariamente plantear prescripciones en general, no para la totalidad sino para la mayoría de los casos. De hecho, el juicio del legislador -observa Aristóteles- no está destinado a la interpretación del caso particular, sino que atañe al futuro y a lo universal (Rhet. $1354 \mathrm{~b} 5$ ss.). Por lo tanto, en todos los casos en que las palabras no definen el delito (Rhet. 1374a 1 ss.), el juez es el único intérprete discrecional de lo que el legislador no ha aclarado respecto al caso en particular. Es pues su juicio el que define lo díkaion, del cual el juez es el único árbitro ${ }^{68}$. Ese juicio, si se adecua a la equidad, tendrá que atender al propósito del legislador (Rhet. $1374 \mathrm{~b} 12$ ss.) y podrá determinar una forma de justicia que vaya más allá de la ley escrita (Rhet. 1374a 28-29).

63. Faraguna, 2007, p. 92 ss.

64. Brunschwig, 1996, p. 117; Saunders, 2001, pp. 80 ss.; Campeggiani, 2009, p. 300.

65. Reseña de fuentes y estudios en Poddighe, 2014, pp. 277-303.

66. Cf. Carey, 1994 y 1996; Sancho Rocher, 2007, pp. 163-5; 295-303, 306-313; Poddighe, 2014, p. 271; GRIFFITH-Williams, 2012 y 2016.

67. Sundahl, 2009, p. 483; Canevaro, 2018.

68. Rhet. 1374 a 28 ss.; Brunschwig, 1996, p. 139; Carey, 1996, p. 42; Poddighe, 2018b. 
La solución que Aristóteles propone para el problema de una definición no pormenorizada del delito en el texto de la ley es, por lo tanto, la equidad como la base del juicio del juez. Se sirve de la expresión "con el mejor espíritu"69. El mejor espíritu interviene en los diferentes casos en que la ley escrita no es adecuada para la causa: sea porque es "contraria al caso", sea porque "alguna vez la ley es contraria a una ya aprobada o incluso a sí misma", sea porque es "ambigua", sea porque "las condiciones bajo las cuales se estableció la ley ya no subsisten". En todos estos casos, obrar "con el mejor espíritu significa precisamente eso: no servirse en exclusividad de las leyes escritas" (Rhet. 1375a 29-1375b 20). La base del juicio ha de ser en estos casos la equidad y corresponde al esfuerzo del juez por comprender la voluntad del legislador y por razonar mediante la valoración de criterios como la probabilidad o la verosimilitud (Rhet. 1376a 18, 1402b 32-33).

Por otro lado, dice Aristóteles, el mejor espíritu interviene también en el caso de que la ley escrita sea adecuada para la causa. En este caso "hay que decir que el mejor espíritu no sirve para pronunciar sentencias al margen de la ley (algo que ningún orador podría afirmar en el tribunal) ${ }^{70}$, sino para que no haya perjurio, si es que se desconoce lo que dice la ley" (Rhet. 1375b 17 ss., cfr. Dem. 23.96-97).

Esta aclaración aristotélica se justifica en el contexto de una reflexión sobre la gnōmé de los jueces como principio capaz de permitir el funcionamiento del sistema de jurados populares, incluso en el caso de que la ignorancia del juez lo lleve a no reconocer el significado de la ley, es decir, a no interpretar su dictado de la mejor manera ${ }^{71}$.

Con esto llegamos a la cuestión central: ¿cómo garantizar la legitimidad de un sistema como el de los jurados populares en ausencia de leyes pormenorizadas y jueces profesionales?

Dado que los límites de las leyes positivas son evidentes, pues no siempre son capaces de decidir el caso concreto, ¿qué puede guiar al juez con respecto a la necesidad de determinar el caso de manera justa, es decir, aplicando -cuando sea necesario-, una "forma de justicia que va más allá de la ley escrita" (Rhet. $1374 a 26$ ss.)?

La respuesta de Aristóteles es que el juez debe admitir entre las bases de su juicio también las leyes no escritas, siempre que se trate de leyes

69. Sobre la superposición de ese concepto (Rhet. 1375a 30-31) con la gnōmè dikaiotáté al que se hace referencia en el juramento heliástico, cf. Talamanca, 1994, pp. 45 ss., MirHADY, 2007, pp. 50, 54, 229 n. 16.

70. SAUNDERS 2001, pp. 78-80; LANNI, 2006, p. 73.

71. Talamanca, 1994, p. 49. 
capaces de obrar en armonía con la politeía, de expresar el èthos constitucional al que debe ajustarse la sentencia.

Ahora bien, los historiadores de las instituciones jurídicas áticas han considerado con razón la posibilidad de que las leyes no escritas constituyeran la base del juicio de los jurados ${ }^{72}$. El hecho de «juzgar" lo que es justo y lo que es injusto apelando a un idem sentire que no solo es producto de las leyes escritas, sino de los valores expresados por la politeía en su conjunto, es el sello distintivo del carácter de los atenienses que Pericles exalta con el mayor énfasis en su Epitafio (Thuc. 2.37). Es solo a través de la manifestación de ese idem sentire como la institución judicial puede reafirmar la concepción de lo justo que la comunidad ha decidido por sí misma ${ }^{73}$. Además, la mentalidad ateniense parece haber admitido la posibilidad de esta interacción no solo en relación con una "Sanción social" genérica, sino como una práctica judicial. Por ejemplo, Lisias recuerda que Pericles aconsejaba basarse en el juicio contra quienes incurrían en el delito de impiedad (asébeia), no solo en los nómoi gegramménoi sino también en los ágraphoi $i^{74}$. Y también Demóstenes reconoce la interacción sinérgica entre tà ágrapha nómima y nómoi gegramménoi como un rasgo distintivo de la politeia ateniense, pero al mismo tiempo como regla que se aplicaba en los juicios por homicidio voluntario decididos por el Areópago ${ }^{75}$. Estas normas -observa Platón- que son "llamadas por la mayoría leyes no escritas [tà ágrapha nómima]" son los "vínculos de toda constitución": se encuentran "entre todas las leyes establecidas por escrito y que están vigentes" $\mathrm{y}$ "mantienen envueltas las leyes escritas entonces con todos los medios útiles para preservarlas" (Plat. Nom. 793a-c).

Es esta interacción la que evoca Aristóteles cuando reflexiona sobre el papel de las leyes no escritas como base del juicio de los jueces ${ }^{76}$. El contenido amplio y genérico de las disposiciones escritas obliga a que las leyes no escritas obren dentro de las escritas, proporcionando contenidos y cánones interpretativos. Por lo tanto, es central en la reflexión aristotélica esta relación dialéctica entre los dos tipos de nómos en el debate procesal, en el que lo importante es establecer de qué lado está lo díkaion ${ }^{77}$. En la base del juicio de equidad se halla la aportación de la ideología dominante a lo que es justo y equitativo.

72. MaFFI, 1990, pp. 71-77. 948.

73. Wallace, 2006, pp. 424-5; Harris, 2006, pp. 53-7; Sancho Rocher, 2007-2008, p.

74. Lys. 6.10; Poddighe, 2014, 58 ss.

75. Dem. 23.70; Talamanca, 2008, pp. 40-4.

76. Cf. Collins, 2006, pp. 142-3; SANCHO RoCHer, 2007-2008.

77. MAFFI, 1983 , p. 254. 
La contribución de la reflexión aristotélica sobre el tema es particularmente relevante, y está bien valorada ${ }^{78}$, aunque quizás no adecuadamente reconocida por la generalidad de los historiadores del derecho, lo que a menudo ha llevado la discusión a temas que para Aristóteles no son objeto de reflexión. Por ejemplo, a Aristóteles no le interesa argumentar la idea de que las leyes no escritas constituyan bases de juicio mejores o de mayor autoridad que las escritas ${ }^{79}$, por más que en ciertos casos esta posibilidad puede verificarse ${ }^{80}$, e incluso si el recurso retórico que afirma esta prioridad es abordado por Aristóteles (Rhet. 1375a 28-35). Tampoco el problema del contraste entre las leyes escritas y no escritas como base del juicio del juez es relevante en su reflexión ${ }^{81}$. Lo que le interesa es enfatizar la oportunidad de que la gnōmé del juez sea lo más conforme posible al èthos de la politeía y, por lo tanto, capaz de aplicar las leyes (escritas y no escritas) que son el producto de "esa" politeía. Lo que cimienta la gnōmè deben ser las leyes particulares de la ciudad, los nómoi idio ${ }^{82}$.

La relevancia de esta categoría de nómoi se reconoce en el contexto del análisis reservado a las relaciones entre nómoi y politeía $a^{83}$. Vale la pena reiterar las características esenciales de la reflexión aristotélica.

Las leyes particulares son aquellas "por las que se gobierna cada ciudad", mientras que las leyes comunes son aquellas "sobre las que parece haber un acuerdo unánime en todos" (Rhet. 1368b 7-9).

La aclaración decisiva para entender qué distinción interesa a Aristóteles se realiza poco después, cuando se afirma que entre las leyes particulares hay tanto leyes escritas como no escritas (Rhet. 1373b 4-8). En efecto, observa Aristóteles: «llamo ley, de una parte, a la que es particular, y de otra, a la que es común, ley particular es la que ha sido definida por cada pueblo en relación consigo mismo, y es unas veces no escrita y otras escrita" (ibid.). Un poco después, resulta aún más claro que la ley particular puede ser escrita o no escrita. Ahí, en efecto, Aristóteles recurre a la perífrasis "ley particular y escrita" (Rhet. 1374a 26-27) ${ }^{84}$.

78. Maffi, 1990, p. 77; 2007, pp. 197-9; HaRRIs, 2013, pp. 31-2.

79. YACK, 1993, pp. 179, 181, 186.

80. Pol. 1287b 5-8, con Mirhady, 2006, p. 11; Sancho Rocher, 2007, p. 153; Ventura, 2009, pp. 80-1.

81. Una cuestión que en cambio promueve la discusión de los historiadores del derecho sobre el testimonio aristotélico (Talamanca, 2008, pp. 31-7, 98-101).

82. MAFFI, 1990, p. 77.

83. Maffi, 1990; Poddighe, 2014, pp. 54-61.

84. Schroeder, 1981, p. 22; Poddighe, 2014, pp. 54-61. 
Por lo tanto, mientras que la ley común es "conforme a la naturaleza" y por lo tanto "parece ser reconocida por todos", la ley particular es la que "ha sido definida por cada pueblo en relación consigo mismo".

Ahora bien, con respecto a la ley natural, debe notarse que, en la práctica judicial, el ágraphos nómos es válido como costumbre y no como derecho natural. La invalidación de una ley escrita en nombre de una ley natural es, de hecho, un argumento del que no se halla constancia de manera significativa y esto confirma que, desde el punto de vista de la historia de las fuentes del derecho, es el ágraphos nómos como costumbre lo que debe considerarse ${ }^{85}$. En cuanto costumbre, el ágraphos nómos es el producto de la politeía. Por ello es relevante, en opinión de Aristóteles, que la gnōmé del juez se apele a esas leyes: en virtud de la relación que existe entre una politeía y su nómoi, entre una politeía y su dikaion ${ }^{86}$. El juez debe determinar la sentencia justa no apelándose a una ley universa ${ }^{87}$, sino a una ley particular, tanto escrita como no escrita, dado que es coherente con su politeia (Rhet. 1373b 4-8, 1374a 26-27) ${ }^{88}$. Es el conocimiento de los nómoi que son expresión de la politeía lo que debe guiar al juez, porque esos nómoi pueden llevarlo a reconocer la voluntad del legislador y, por lo tanto, el "espíritu de la ley", incluso cuando su formulación no esté lo suficientemente pormenorizada ${ }^{89}$. Este es el espacio operativo del mejor juicio o gnömè del juez que obra de acuerdo con los principios de la epieíkeia. Esta gnōmè, basada en las leyes particulares, compensa la criticidad debida al carácter genérico de la ley escrita (Rhet. 1374a 26-b23; EN 1137b 23-24, 1141b 21-22; Pol. 1269a 10-11, 1286a 10-11) ${ }^{90}$. Es en este marco donde opera la epieíkeia, en sinergia con la ley y con el objetivo de adaptar la universalidad de la ley positiva al caso particular, para resolver la tensión entre ley universal y caso particular ${ }^{91}$, por ejemplo, dando el peso adecuado a las circunstancias "atenuantes" ${ }^{92}$.

85. MafFi, 1983, p. 256. Al igual que Brunschwig, 1996, p. 144; quien observa que Aristóteles no evoca el derecho natural como base del juicio de equidad y que el ágraphos nómos deben entenderse como "customs, mores, specific moral intuition of a given social and political group". Sobre el asunto cf. Talamanca, 2008, pp. 103 ss.

86. Poddighe, 2014, pp. 46-61; Sancho Rocher, 2007, p. 149 nn. 11-12; Ventura, 2009.

87. BrunsChwig, 1996, p. 148.

88. Brunschwig, 1996, pp. 143-4; Harris, 2013, p. 32. De otro parecer es Talamanca (1984, pp. 338-42), que identifica el koinós nómos con el ágraphos nómos.

89. BRunsChWig, 1996, p. 150; Vega, 2013, p. 199.

90. SANCHO ROCHER, 2007, pp. 154-5.

91. Tensión que Platón había dejado sin resolver (BRunschwig, 1996, pp. 118, 130).

92. BRunschwig (1996, pp. 150-1) subraya la oportunidad de que el juez tuviera en cuenta las intenciones en lugar del resultado de una acción, el espíritu del legislador en 
La mejor facultad de juicio, fundada en la epieíkeia, no es, por lo tanto, un instrumento para hacer caso omiso de la ley, sino que es funcional para interpretar su espíritu o, en cualquier caso, para guiar al jurado en el ejercicio de la función jurisdiccional ${ }^{33}$.

Como conclusión, vamos a resumir los principios constitutivos del pensamiento político sobre el papel de los jueces populares que dan con su sentencia un juicio conforme con el concepto de justicia global.

En primer lugar, se halla la idea de que una politeía debe formar ciudadanos educados de acuerdo con sus principios y, por lo tanto, capaces de juzgar siguiendo el sentido común (epieíkeia) tanto en los casos no determinados por las leyes escritas como en los casos que, aunque contemplados por las leyes escritas, requieren un ejercicio de sentido común "que va más allá de la ley escrita" (Rhet. 1374a 28-29). En todos esos casos, una educación coherente con el èthos de la politeía y el conocimiento de los nómoi de la polis permite a los ciudadanos ejercer su mejor facultad de juicio (Rhet. 1365b 21-1366a 21) ${ }^{94}$, es decir, reconocer el "espíritu de la ley", incluso cuando su formulación no está lo suficientemente detallada ${ }^{95}$.

Es midiéndose con los límites de la ley -es decir, al abordar la criticidad debida a la naturaleza genérica de la ley escrita- cuando se especifica el espacio operativo del mejor juicio o gnōmè que debe funcionar de acuerdo con los principios de la epieíkeia (sin abusar de esta) ${ }^{96}$. Dado que la epieíkeia obra en sinergia con la ley y con el objetivo de adaptar la universalidad de la ley positiva al caso particular, a través de la mejor capacidad de juicio, los ciudadanos interpretan lo que el legislador no ha aclarado con respecto al caso particular. Es pues su juicio lo que define lo dikaion de la polis (Rhet. 1374a, 28 ss.) ${ }^{97}$.

lugar de la letra de la ley, toda una vida y no un solo episodio. Cfr. Campeggiani, 2009, p. 308; HARRIS, 2013, p. 48.

93. Maffi (1990, p. 77) considera que, dada la incapacidad del conjunto de las leyes escritas para "constituir un código", la costumbre fue "reconocida como un marco regulatorio" y encontró "una aplicación a nivel judicial tanto directa como indirectamente como herramienta para la interpretación de la ley escrita"; en contra se muestra Talamanca (2008, p. 98 y n. 263). Sobre la discusión cf. Poddighe, 2014, pp. 54-61, y Poddighe, 2018b.

94. Poddighe, 2014, pp. 265-266.

95. BRunsChWig, 1996, p. 150.

96. La reflexión de Aristóteles admite esta posibilidad en Rhet. 1375a 27-b 25. Sobre esta "interpretación cautiva", cf. BERTELLI, 2015, pp. 30-31. Es objeto de discusión el que este abuso fuera frecuente en los tribunales atenienses: cf. HarRIs, 2013, pp. 274-301; PODDIGHE, 2014, pp. 275-303.

97. Brunschwig, 1996, p. 139. Cf. también Hamburger, 1951, pp. 89-105; Aubenque, 1965, p. 112 y p. 156; Moraux, 1965, pp. 156-157; Wormuth, 1978, pp. 18-19; Frank, 2005, 
Al ejercer este papel, los ciudadanos deberán admitir también leyes no escritas entre las bases de su juicio ${ }^{98}$, siempre y cuando sean leyes capaces de operar en sintonía con la politeía $a^{99}$, de expresar el éthos constitucional al que debe conformarse la decisión sobre los casos particulares ${ }^{100}$. La posibilidad de que las leyes no escritas constituyeran la base del juicio de los jurados ha sido acertadamente considerada por los historiadores de las instituciones jurídicas áticas ${ }^{101}$ y es esa misma interacción la que evoca Aristóteles cuando reflexiona sobre el "mejor juicio" basado en el sentido común ${ }^{102}$.

En el marco de la interacción entre nomos general que adaptar a los casos particulares y epieíkeia, se especifica la tarea confiada a los ciudadanos: interpretar la ley y afirmar con el mejor juicio la noción de lo «justo político" (lo díkaion). Lo justo político incorpora lo justo legal (su componente esencial) pero es más amplio y permite un cierto movimiento entre leyes escritas y no escritas ${ }^{103}$. A través del juicio más justo, los ciudadanos realizan en los tribunales la tarea que la reflexión aristotélica les confía en el ámbito de la aplicación y adaptación de las leyes a los casos particulares.

\section{Bibliografía}

Accattino, P.: "Aristotele. La Politica. Commento al libro III", en Accattino, P. y Curnis, M. (a cura di): Aristotele. La Politica. Volume III (libro III). Roma, 2013, pp. 147-239.

pp. 114-115; Miller, 2007, p. 101; Accattino, 2013, p. 231; Harris, 2013, pp. 274-301; Bertelli, 2015, pp. 27-28; Poddighe, 2018b, pp. 160, 172-173.

98. Cf. Brunschwig, 1996, p. 140; Prado, 2004, p. 207; Campeggiani, 2009, pp. 293-300; Poddighe, 2014, pp. 265-274; Bertelli, 2015, p. 28.

99. Cf. Brunschwig, 1996, p. 140; Prado, 2004, p. 207; Campeggiani, 2009, pp. 293-300; Harris, 2013, pp. 276-285; Poddighe, 2014, pp. 265-274; Bertelli, 2015, p. 28.

100. Cf. Brunschwig, 1996, p. 151; Thomas, 2005, pp. 42 y 59; Harris, 2013, pp. 274301; Poddighe, 2014, pp. 59, 273-274, 297; Bertelli, 2015, pp. 27-31. Para la epieikeia en la que se basa la argumentación de los oradores cf. HarRIs, 2013, pp. 285 ss.

101. Cf. Maffi, 1990, pp. 71-77. Para la idea aristotélica de que los cimientos de la gnòmè deben ser todas las leyes de la ciudad, cf. también Poddighe, 2014, pp. 269-272.

102. La contribución de la reflexión aristotélica sobre el tema es particularmente relevante y bien valorada por los estudiosos (MAFFi, 1990, p. 77; 2007, pp. 197-9; BeARzOT, 2013, pp. 85-98; Harris, 2013, pp. 276-285). Cf. también Poddighe, 2014, pp. 275-303.

103. Cf. BerTelli, 2015, pp. 27-28 en EN 1137b 12-33. Sobre la interacción entre lo justo legal y político en la Ética a Nicómaco, cf. PodDighe, 2014, pp. 47-53; 2016. 
Aubenque, P.: Théorie et pratique chez Aristote, en La Politique d'Aristote, "Entretiens sur l'Antiquité classique", XI. Vandoeuvre-Genève, 1965, pp. 97-114.

Aubenque, P.: "La loi selon Aristote", Archives de Philosophie du Droit, 25. 1980, pp. 147-157.

Bearzot, C.: "La gnome del giudice nell'oratoria attica", en Bearzot, C. y Vimercati, E. (a cura di): La giustizia dei Greci tra riflessione filosofica e prassi giudiziaria. Milano, 2013, pp. 85-98.

Bearzot, C.: "Aristotele, EN V: tra prassi politica e giudiziaria e riflessione teorica", Antiquorum Philosophia, 10, 2016, pp. 93-107.

Bertelli, L.: "Metabolē politeiōn", Filosofia Politica, 3/2, 1989, pp. 275-326.

Bertelli, L.: "La giustizia di Aristotele", en Harris, E. M. y Canevaro, M. (eds.): Oxford Handbook of Ancient Greek Law (Oxford Handbooks Online), 2015.

BERTRAND, J. M.: “Àpropos de la "Rhetorique" d'Aristote (i 1373b1-1374b23), analyse du processus judiciaire (to epigramma - to enklema)", Dike, 5, 2002, pp. 161-85.

Bodéüs, R.: "Law and Regime in Aristotle", en Lord, C. y O'Connor, D. K. (eds.): Essays on the Foundation of Aristotelian Political Science. Berkeley (CA), 1991, pp. 234-48.

Boegenold, A.: "Resistance to Change in the Law at Athens", en OBer, J. y Hedrick, C. (eds.): Demokratia. A Conversation on Democracies, Ancient and Modern. Washington D. C., 1996, pp. 203-14.

Brunschwig, J.: "Du mouvement et de l'immobilité de la loi", Revue Internationale de Philosophie, 133-134, 1980, pp. 512-540.

Brunschwig, J.: "The Aristotelian Theory of Equity", en Frede, M. y STRIKER, G. (eds.): Rationality in Greek Thought. Oxford, 1996, pp. 115-55.

Bullen, P.: "Lawmakers and Ordinary People in Aristotle", en Rubin, L. G. (ed.): Justice v. Law in Greek Political Thought. London-Lanham-New York, 1997, pp. 229-241.

Camassa, G.: "I Greci davanti al problema del mutamento", Quaderni di storia, 57, 2003, pp. 147-172.

CAMmaK, D.: Rethinking Athenian Democracy Cambridge (MA), 2013.

Campeggiani, P.: "La nozione di epieíkeia in Aristotele: il giudice e la norma", Il Pensiero Politico, 42, 2009, pp. 1-23.

Canevaro, M.: "Aristotele. La Politica. Commento al libro IV (14-16)", en Guagliumi, B. y Curnis, M. (a cura di): Aristotele. La Politica. Volume IV (libro I). Roma, 2014, pp. 279-377.

Canevaro, M.: "Laws Against Laws: the Athenian Ideology of Legislation", en Carey, C., Giannadaki, I. y Griffith Williams, B. (eds.): Use and Abuse of Law in Athenian Courts. Leiden, 2018, pp. 271-292. 
CAREY, C.: "Artless' proofs in Aristotle and the Orators", BICS, 39, 1994, pp. 95-106.

Carey, C.: "Nomos in Attic Rhetoric and Oratory", JHS, 116, 1996, pp. 3346.

Coluins, T.: "Aristotle's Legislative Science", en Rubin, L. G. (ed.): Justice v. Law in Greek Political Thought. London-Lanham-New York, 1997, pp. 213-227.

Crifò, G.: Civis. La cittadinanza tra antico e moderno. Roma-Bari, 2005.

Frank, J.: A Democracy of Distinction: Aristotle and the Work of Politics. Chicago, 2005.

Griffith-Williams, B.: "Oikos, family feuds and funerals: Argumentation and evidence in Athenian inheritance disputes", CQ, 62, 2012, pp. 145-162.

Griffith-Williams, B.: "Rational and emotional persuasion in Athenian inheritance cases", en SANDERS, E. y Johncock, M. (eds.): Emotion and persuasion in classical antiquity. Stuttgart, 2016, pp. 41-55.

FARAGUNA, M.: "Tra oralità e scrittura: diritto e forme della comunicazione dai poemi omerici a Teofrasto", Etica E Politica, 9, 2007, pp. 75-111.

FARenga, V.: Citizen and Self in Ancient Greece. Individuals Performing Justice and the Law. Cambridge, 2006.

Hamburger, M.: Morals and Law: The Growth of Aristotle's Legal Theory. New Haven, 1951.

Hansen, M. H.: "Nomos and Psephisma in Fourth-Century Athens", GRBS, 19, 1978, pp. 315-330.

Harris, E. M.: "Open Texture in Athenian Law”, Dike, 3, 2000, pp. 27-79.

Harris, E. M.: The Rule of Law in Action in Democratic Athens. Oxford, 2013.

Horn, C.: "Law, Governance, and Political Obligation", en Deslauriers, M. y P. Destrée, P. (eds.): The Cambridge Companion to Aristotle's Politics. Cambridge, 2013, pp. 223-246.

Johnstone, S.: Disputes and Democracy. The Consequence of Litigation in Ancient Athens. Austin (TX), 1999.

KasAI, Y.: "A Space for epieíkeia in Greek Law", en THür, G. (ed.): Symposion 2009: Vorträge zur griechischen und hellenistischen Rechtsgeschichte. Wien, 2010, pp. 117-26.

Kraut, R.: Aristotle: Political Philosophy. Oxford, 2002.

LANe, M.: "Claims to rule: the case of the multitude", en Deslauriers, M. y Destrée, P. (eds.): The Cambridge Companion to Aristotle's Politics. Cambridge, 2013, pp. 247-74.

Lanni, A.: Law and Justice in the Courts of Classical Athens. Cambridge, 2006. 
LODDO, L.: Solone demotikotatos. Il legislatore e il politico nella cultura democratica ateniese, Milano, 2018.

López Barja De Quiroga, P. M.: "El jurado y la democracia ateniense", Revista de Occidente, 174, 1995, pp. 138-152.

López BARja DE Quiroga, P. M.: "Aristóteles: el gobierno de los mejores" en Sancho Rocher, L. (ed.): Filosofía y democracia en la Grecia antigua. Zaragoza, 2009, pp. 199-228.

LORD, C.: Aristotle's Politics, Chicago-London, 2013.

MacDowell, D. M.: The Law in Classical Athens. London, 1978.

Maffi, A.: «M. Bretone - M. Talamanca, Il diritto in Grecia e a Roma, RomaBari 1981", QS, 17, 1983, pp. 245-260.

Maffi, A.: "La consuetudine nella Grecia arcaica e classica", La coutume. Recueils de la Societé Jean Bodin pour l'bistoire comparative des institutions, 51, 1990, pp. 73-79.

MafFI, A.: «Funzione giurisdizionale e regimi politici nella Grecia arcaica e classica", en Cataldi, S. (a cura di): Poleis e Politeiai. Esperienze politiche, tradizioni letterarie, progetti costituzionali. Alessandria, 2004, pp. 305-14.

Maffi, A.: "Risposta a Robert W. Wallace", en Cantarella, E. (hrsg.): Symposion 2005. Vorträge zur grieschischen und bellenistischen Rechtsgeschichte (Salerno, 1-18 September 2005). Stuttgart, 2007, pp. 197-200.

Mayhew, R.: "Rulers and Ruled", en Anagnostopoulos, G. (ed.): $A$ Companion to Aristotle. Oxford, 2009, pp. 526-539.

Meyer-Laurin, H.: Gesetz und Billigkeit im attischen Prozess. Weimar, 1965. Millett, P.: "Sale, Credit and Exchange in Athenian Law and Society", en Cartledge, P., Millett, P. y Todd, S. (eds.): Nomos. Essays in Athenian Law, Politics and Society. Cambridge, 1990, pp. 167-194.

Mirhady, D. C.: "Aristotle and the Law Courts", Polis, 23, 2, 2006, pp. 1-17. Mirhady, D. C.: "The Dikasts' Oath and the Question of Facts", en A. H. Sommerstein, J. Fletcher (eds.), Horkos. The Oath in Greek Society, Exeter 2007

Moraux, P.: "Quelques apories de la politique et leur arrière-plan historiques", La Politique d'Aristote. Entretiens sur l'Antiquité classique, XI, 1965, pp. 126-148.

Miller, Jr., F. D.: "Aristotle's philosophy of law", en Miller, F. D. y Biondi, C. A. (eds.): A History of the Philosophy of Law from the Ancient Greeks to the Scholastics. Dordrecht, 2007, pp. 79-110.

O'Neil J. L.: "Was the Athenian Gnome Dikaiotate a Principle of Equity?", Antichthon, 35, 2001, pp. 20-9. 
Osborne, R.: "When Was the Athenian Democratic Revolution?", en S. D. GOLDHILLD, S. D.

Osborne, R. (eds.). Rethinking Revolutions Through Ancient Greece. Cambridge, 2006, pp. 10-28.

Osborne, R.: Athens and Athenian Democracy. Cambridge, 2010.

Ostwald, M.: From Popular Sovereignty to the Sovereignty of Law. Berkeley (CA), 1986.

Paoli, U. E.: Studi sul processo attico. Padova, 1933.

Pelloso, C.: "L' है $\varphi \varepsilon \sigma ı \varsigma$ al tribunale popolare in diritto processuale ateniese: "impugnazione", "rimessione" o "tertium datur"?", Index, 45, 2017, pp. 518-556.

Pezzoli, F.: "Aristotele. La Politica. Commento al libro II", en Pezzoli, F. y Curnis, M. (a cura di): Aristotele. La Politica. Volume II (libro II). Roma, 2012, pp. 171-411.

Pezzoli, F.: "La figura del legislatore nella Politica di Aristotele", RDE, IV, 2014, pp. 167-178.

Poddighe, E.: Aristotele, Atene e le metamorfosi dell'idea democrática. Roma, 2014.

Poddighe, E.: "Arist. Ath. Pol. 9.2 e la regola del giudizio globale sui politika. Considerazioni sul metodo storico aristotelico", en BEARzot, C., Canevaro, M., Gargiulo, T. y Poddighe, E. (eds.): Athenaion Politeiai tra storia, politica e sociologia: Aristotele e Ps-Senofonte, Atti del Convegno Internazionale di Studi (Cagliari 10-12 Maggio 2017). Milano 2018a, pp. 147-174.

Poddighe, E.: "Aristotele e la legge: il tema del mutamento", en BiscotTi, B. (ed.): Kállistos Nómos. Scritti in onore di Alberto Maffi. Torino 2018b, pp. 153-176.

Prado, M.: "Epieikeia y razionamento jurídico en Aristóteles", Persona y Sociedad, 17.2, 2003, pp. 205-226.

Rhodes, P. J.: A Commentary on the Aristotelian Athenaion Politeia. Oxford, 1981.

Rhodes, P. J.: "The Athenian Code of Laws, 410-399 B.C.", JHS, 111, 1991, pp. 87-100.

Rhodes, P. J.: "Appeals to the Past in Classical Athens", en Herman, G. (ed.): Stability and Crisis in the Athenian Democracy. Stuttgart, 2011, pp. 13-30.

SÁNCHEZ MADRID, N.: "Democracia, concordia y deliberación pública en la Política de Aristóteles", Logos. Anales del Seminario de Metafísica, 51, 2018, pp. 35-56.

SAncho Rocher, L.: "Justice et légalité chez Aristote et dans la pratique démocratique", RIDA, 54, 2007, pp. 143-80. 
SANCHO Rocher, L.: "Condicionamientos democráticos y sistema judicial ateniense", Veleia, 24-25, 2007-2008, pp. 939-953.

SAmaras, Th.: Plato on Democracy. New York, 2002.

SAunders, T. J.: "Epieikeia: Plato and the Controversial Virtue of the Greeks", en Lisi, F. L. (ed.): Plato's Laws and Its Historical Significance. Sankt Augustin, 2001, pp. 65-93.

Schroeder, D.: "Aristotle on Law", Polis: The Journal for Ancient Greek Political Thought, 4, 1981, pp. 17-31.

Schütrump, E.: Aristoteles, Politik Buch II/III. Berlin-Darmstadt, 2001.

SHEAR, J. L.: Polis and Revolution. Responding to Oligarchy in Classical Athens. Cambridge, 2011.

Simpson, P. L.: A Philosophical Commentary on the Politics of Aristotle. University of North Carolina Press, Chapell Hill-London, 1998.

Sundahl, M. J.: "The Living Constitution of Ancient Athens: A Comparative Perspective on the Originalism Debate", The John Marshall Law Review, 42, 2009, pp. 463-504.

TAlamancA, M.: "Politica, equità e diritto nella pratica giudiziaria attica", en Dimakis, P. D. (ed.): Mneme G. A. Petropoulos, II. Athens, 1984, pp. 333-56.

Talamanca, M.: "Il diritto in Grecia", en Talamanca, M. y Bretone, M.: Il diritto in Grecia e a Roma. Roma-Bari, 1994 (2. ${ }^{a}$ ed.), pp. 5-89.

TALAMANCA, M.: "Ethe e nomos agraphos nel Corpus oratorum atticorum", en Bove, L. (a cura di): Prassi e diritto. Valore e ruolo della consuetudine. Napoli, 2008, pp. 3-104.

Thomas, R.: "Writing Law, and Written Law", en Gagarin, M. y Cohen, D. (eds.): The Cambridge Companion to Ancient Greek Law. CambridgeNew York, 2005, pp. 41-60.

TodD, S. C.: "Lysias against Nikomachos: The Fate of the Expert in Athenian Law", en Foxhall, L. y LewIs, A. D. (eds.): Greek Law in Its Political Setting: Justifications not Justice. Oxford, 1996, pp. 122-36.

VenturA, D.: Giustizia e costituzione in Aristotele. Milano, 2009.

VOLONAKI, E.: "The Re-publication of the Athenian Laws in the Last Decade of the Fifth Century BC", Dike, 4, 2001, pp. 137-67.

VergniÈres, S.: Éthique et politique chez Aristote: physis, êthos, nomos. Paris, 1995.

Wallace, R. W.: The Areopagos Council to 307 B.C. Baltimore (MD), 1989.

Wallace, R. W.: "Law and Rhetoric: Community Justice in Athenian Courts", en Kinzl, K. H. (ed.): A Companion to the Classical Greek World. Oxford, 2006, pp. 416-31.

Wexler, S. e Irvine, A.: "Aristotle on the Rule of Law", Polis: The Journal for Ancient Greek Political Thought, 23.1, 2006, pp. 116-138. 
Wormuth, F. D.: "Aristotle on Law", en Nelson, D. H. y SkLar, R. D. (eds.): Essays in Law and Politics. Port Washington, NY, 1978, pp. 14-26.

YACK, B.: The Problems of a Political Animal: Community, Justice, and the Conflict in Aristotelian Political Thought. Berkeley-Los AngelesLondon, 1993.

ZacCARINI, M.: "The Fate of the Lawgiver. The Invention of the Reforms of Ephialtes and the Patrios Politeia", Historia, 40, 2018, pp. 1-18. 
